\title{
LA QUINUA (Chenopodium quinoa Willd.) ALTERNATIVA DE SEGURIDAD ALIMENTARIA PARA ZONAS DESÉRTICAS
}

\author{
QUINOA (Chenopodium quinoa Willd.) ALTERNATIVE OF FOOD SAFETY FOR \\ DESERT AREAS
}

\author{
'Nivardo Núñez Torreblanca
}

\begin{abstract}
RESUMEN
La quinua (Chenopodium quinoa Willd.) es una especie originaria de los andes Sudamericanos, que ha mostrado respuestas fisiológicas que son características de especies que toleran sequia y sales, por lo que constituye una alternativa de alto valor para obtención de proteinas y fibra de alta calidad en áreas donde el agua y las sales del suelo son restrictivos para que prosperen otros cultivos. Aproximadamente, casi en la mitad de la superficie del planeta están presentes las zonas áridas. El rasgo característico de estas zonas es que la sequia es un evento regular, con presencia también de altas temperaturas, con diferentes grados de salinidad de los suelos. Se estima que un quinto de la población mundial vive en estas zonas. En el caso particular del Perú el 90 por ciento de la población nacional habita estas zonas. En general, el agua es un recurso escaso, por lo que es prioridad el estudio de plantas alimenticias que toleren las condiciones adversas de estas áreas.
\end{abstract}

Palabras Clave: Fotosíntesis, sequía, conductancia, salinidad, aridez, quinua.

\section{ABSTRACT}

Quinoa (Chenopodium quinoa Willd.) as a species of South American Andes, which has shown physiological responses that are characteristic of species that tolerate drought and salt, which is a high-value alternative for obtaining proteins and high fiber quality in areas where water and soil salts are restrictive for other crops flourish. In approximately almost half of the planet's surface arid areas. The characteristic feature of these areas is that drought is a regular event, with the presence also of high temperatures with varying degrees of soil salinity. An estimated one fifth of the world population lives in these areas. In the particular case of Peru 90 percent of the national population live in these areas. In general, water is a scarce resource, so it is priority the study of food plants that tolerate adverse conditions in these areas.

Keywords: photosynthesis, drought, conductivity, salinity, aridity, quinoa.

\section{INTRODUCCIÓN}

La quinua (Chenopodium quinoa Willd.) es una especie cuyo centro de origen se considera en los andes Sudamericanos (Cárdenas, 1994). La mayor variabilidad de genotipos y de progenitores silvestres se encuentran en las proximidades del Lago Titicaca (Perú - Bolivia) y la mayor diversidad se ubica entre Potosí en Bolivia y Sicuani en Perú (Mujica et al., 2000).

A partir de mediados de la década de los años 50 del siglo XX, los estudios por ampliar los conocimientos sobre esta planta toman mayor impulso y comienzan a realizarse colecciones de ecotipos y accesiones en Perú, Bolivia, Ecuador y Colombia (Lescano, 1994).

La quinua ofrece sobresalientes cualidades nutricionales, contiene entre 13,81 y $21,9 \%$ de proteínas y más aún la calidad de éstas son del tipo albúmina y globulina. El alto valor alimenticio que se le reconoce se debe al contenido de aminoácidos, que se encuentran en el núcleo del grano, ventaja notable sobre el arroz o trigo que los tienen en su exosperma o cáscara. La quinua es considerada como el único alimento vegetal que puede proveer de todos los aminoácidos esenciales, oligoelementos, vitaminas y no contener gluten (FAO. 2011). Este preciado grano andino ha despertado el interés de muchos investigadores, más allá de las fronteras de Sudamérica.

Entre los principales productores de este importante grano, se encuentran Perú y Bolivia, en menores proporciones figuran Estados Unidos (seis por ciento) con una producción de 3000 t, Ecuador y Canadá, pues producen aproximadamente el 10 por ciento de la producción mundial. La superficie cultivada en los países andinos ha experimentado incrementos sucesivos durante los últimos años debido a los requerimientos del mercado. Para el año 2009, el área culti- 
Nuñez, N. La quinua (Chenopodium quinoa Willd.) alternativa de seguridad alimentaria para zonas desćrticas.

vada fue de 83000 ha. La producción en éstos países es del orden de $70000 \mathrm{t}$, de las cuales 40000 corresponden al Perú, 28000 t, a Bolivia, y a Ecuador 746 t (FAO, 2011). Para el caso de Europa la producción es de $210 \mathrm{t}$ (FAO, 2011).

Su cultivo se ha extendido a otras partes del mundo, como el Reino Unido donde se vende en tiendas de comidas saludables, En Dinamarca es consumida por personas que no toleran el gluten de los cereales. Holanda es el país que ha logrado la primera variedad europca con el nombre de "Carmen". La quinua también fue llevada a los Himalayas (Asia), sin embargo no tuvo mayor aceptación por su contenido de saponinas, e inclusive al Tibet (Jacobsen y Risi, 2000). Material genético procedente de Colombia cultivados en Kenya (África) mostraron rendimientos en grano de hasta 4000 $\mathrm{kg} / \mathrm{ha}$, lo que constituye una real alternativa para los problemas de alimentación en aquel continente (Jacobsen, 2003).

El creciente interés por la quinua ha permitido que adquiera importancia cconómica, de tal manera que hoy es un cultivo de exportación, motivo por el que, en el caso del Perú, en los últimos años, se le viene cultivando en la costa sur (Tacna), donde tradicionalmente predominaba el olivo y otros cultivos hortícolas.

Se han realizado diversas investigaciones para conocer sus atributos, referente a su valor alimenticio, capacidad productiva con diferentes tratamientos, entre ellos, comportamiento en condiciones de estrés hídrico (sequía), salinidad, y otros factores adversos como exceso de humedad, granizadas, frio, calor, vientos; posibilidades de transformación e industrialización, en muchas de las cuales se han encontrado respuestas muy favorables y alentadoras.

Por su plasticidad es capaz de crecer y desarrollar en variadas condiciones ecológicas. Puede prosperar en climas desérticos, calurosos y secos de la costa del Perú, como en aquellos muy frigidos, en otros menos rigurosos como lo valles interandinos, o condiciones de clima subtropical; de manera que se le puede cultivar en cinco de las ocho zonas de vida natural identificadas por Pulgar Vidal (para el Perú), las que se encuentran entre el nivel del mar y los 4000 m.s.n.m. En relación a su distribución, se conoce su adaptación a los diferentes climas andinos (Tapia, 2001). Estudios preliminares en la costa sur del Perú (Tacna) indican que la quinua puede cultivarse por lo menos en diez de los doce meses del año.

Dadas las características descritas, Chenopodium quinoa Willd. se constituye en un cultivo de gran valor, no sólo para las poblaciones donde tradicionalmente se le cultiva, sino para la población mundial, considerando que un importante porcentaje de personas viven en zonas desérticas, donde los principales limitantes para la producción de alimentos, son la carencia del recurso agua y las condiciones de salinidad de los suelos.

En el Perú, las zonas áridas y semiáridas representan un área mayor a los 30 millones de hectáreas, lo que es equivalente a un cuarto del total de la superficie nacional aproximadamente y comprenden toda la costa y parte de la sierra. Estas zonas se encuentran habitadas por el 90 por ciento de la población nacional (UNALM, 1982).

Las zonas desérticas se caracterizan por presentar severas limitaciones para la producción agrícola, precipitaciones en niveles muy bajos, que pueden estar por debajo de los $200 \mathrm{~mm}$ anuales, tal como es el caso de la costa sur del
Perú. Sin embargo, las tasas de evaporación y transpiración son elevadas, lo que da como resultado un déficit permanente de humedad del suelo. Las características descritas anteriormente, también dan por resultado en éstas zonas una alta concentración de sales solubles en el suelo. En estas condiciones desfavorables es que se busca el rendimiento de los cultivos, tanto para cubrir los requerimientos de la población así como para la exportación.

\section{Caracterización de las zonas áridas}

Las zonas áridas se definen como aquellas unidades geográficas y ecológicas o regiones, en donde, debido a una situación de sequía casi permanente, la presencia de especies vegetales es escasa, están condicionadas por elevadas tasas de evaporación, debido a altas temperaturas, baja humedad, vientos muy fuertes, en otros casos pendientes pronunciadas que favorecen la escorrentía y suelos sueltos con poca capacidad de almacenamiento hídrico. Estas características pueden estar acompañadas con diferentes niveles de salinidad. Sin embargo, el término aridez puede resultar ser relativo, si las condiciones de precipitación que en una región son insuficientes, en otra, puede permitir la existencia de algún tipo de vegetación, por lo que pucden identificarse grados de aridez de acuerdo al balance entre las ganancias y pérdidas de humedad (Medrano y Flexas, 2004).

El término sequía indica que se trata de un período con ausencia de precipitaciones, durante el cual, el contenido del agua del suelo se reduce a niveles tales que, por la carencia de humedad, las plantas sufren alteraciones en sus funciones normales. Frecuentemente, aunque no invariablemente, la deshidratación del suelo está relacionada con evaporación intensa, debido a la sequedad del aire y altos niveles de radiación. En zonas áridas, la sequía ocurre de manera regular y prolongada (Larcher, 1995).

En general, en aquellas zonas en que predomina el déficit hídrico durante todo el año las tierras áridas se clasifican como extremadamente áridas o hiperáridas; en tanto ocurra en la mayor parte del año, se clasifican como regiones áridas, o, semiáridas. $\mathrm{La}$ aridez se evalúa sobre la base de las variables climáticas, a la que se ha denominado "índice de aridez", o de acuerdo con la propuesta de la FAO, sobre la base de cuántos días el balance de agua permite el crecimiento de las plantas. El indice de aridez utiliza la relación $\mathrm{P} / \mathrm{ETP}$ (precipitación/evapotranspiración potencial), para clasificar las tierras áridas (FAO, 2007). (Ver tabla 1).

De acuerdo a las estimaciones del UNEP (1997), aproximadamente el 47 por ciento (casi la mitad) de la superficie de la tierra está ocupada por zonas áridas, lo que es equivalente a 6 mil millones y medio de hectáreas aproxima-

Tabla 1. Categorias de tierras áridas según la FAO (1993), clasificación y extensión (PNUMA, 1992)

\begin{tabular}{|c|c|c|c|}
\hline Clasificación & P/ETP & $\begin{array}{l}\text { Precipitación } \\
(\mathrm{mm})\end{array}$ & $\begin{array}{l}\text { Área } \\
(\%)\end{array}$ \\
\hline Hiperáridas & $<0,05$ & $<200$ & 7,50 \\
\hline Áridas & $0,05<\mathrm{P} / \mathrm{ETP}<0,20$ & $\begin{array}{c}<200 \text { (invierno }) \circ \\
<400 \text { (verano })\end{array}$ & 12,1 \\
\hline Scmiáridas & $0,20<\mathrm{P} / \mathrm{ETP}<0,5$ & $\begin{array}{r}200-500 \text { (invicino) o } \\
<400-600 \text { (verano) }\end{array}$ & 17,7 \\
\hline Secas subhúmedas & $0,5<$ P $/$ ETP $<0,65$ & $\begin{array}{l}500-700 \text { (inviemo) o } \\
<600-800 \text { (verano) }\end{array}$ & 9,90 \\
\hline Total & & & 47,2 \\
\hline
\end{tabular}

Fuente: FAO, 2007 
Nuñez, N. La quinua (Chenopodium quinoa Willd.) Alternativa de seguridad alimentaria para zonas desćrticas.

damente, distribuidas en grandes regiones del planeta. De las cuales mil millones de hectáreas son hiperáridas y se extienden mayormente a lo largo de los desiertos del Sahara, Arabia y Gobi. Las regiones áridas, semiáridas, subhúmedas secas ocupan más o menos cinco millones y medio de hectáreas. Las precipitaciones en las regiones áridas varian entre $200 \mathrm{~mm}$ anuales en las áreas con lluvia de invierno, a $300 \mathrm{~mm}$ en las áreas con lluvias de verano. En el año 2000, esta zona estaba habitada por un quinto de la población mundial (FAO, 2007).

Según la ONU (mencionado por ALIDE, 2010), una de cada tres personas en el mundo vive en tierras secas o áridas; dos mil millones de habitantes de tierras secas viven con menos de un dólar al día y sin acceso adecuado al agua; para el año 2030 , se estima que el 50 por ciento de la población mundial estará viviendo en zonas de gran estrés de agua.

\section{Respuestas de la quinua al déficit hídrico}

Se han realizado diversas investigaciones para conocer los mecanismos por los cuales la quinua puede tolerar episodios de sequía. Inicialmente esta capacidad se atribuyó a la morfología de la planta, debido a la presencia de papilas higroscópicas en la cutícula de la hoja o su sistema radicular bastante ramificado (Canahua, 1977).

El estudio de las respuestas fisiológicas permiten un conocimiento más amplio del comportamiento de la quinua en condiciones de estrés hidrico. De esta manera se realizan investigaciones tomando como referencia las fases fenológicas del cultivo.

Jensen et al. (2000) estudiaron las respuestas de la quinua en tres fases fenológicas, en las que el contenido de humedad del suelo, desde capacidad de campo descendió hasta valores extremos de tres y uno por ciento en base a peso. En esas condiciones, la tasa fotosintética en la fase fenológica de ramificación, también descendió hacia niveles de $10 \mu \mathrm{molm}{ }^{2} \mathrm{~s}^{1}$. En las fases de floración y llenado de grano, luego de un rápido descenso, la fotosíntesis fue de cero. De manera similar la conductancia $\left(\mathrm{g}_{\mathrm{H} 2 \mathrm{u}}\right)$ disminuyó a $0,1 \mathrm{molm}^{2} \mathrm{~s}$, en estas circunstancias, el cierre estomatal ocurrió cuando el potencial hídrico foliar llegó a valores inferiores a $-0,9 \mathrm{en} \mathrm{la}$ fase de ramificación, que fue menos negativo con respecto a las fases de floración y llenado de grano en las cuales fue de - 1,1 MPa y se encontró al ta correlación positiva $(\mathrm{r}=0,98)$.

Estas respuestas se encuentran asociadas al comportamiento estomatal en relación a los factores ambientales, grado de deshidratación del suelo y sus efectos sobre la función fisiológica y metabólica de la planta. La alta correlación entre la conductancia y la fotosintesis, obedece a que ambas variables están controladas en alto grado por el cierre y apertura de estomas. El estrés por sequía da lugar al cierre de cstomas lo que provoca la reducción de los niveles de conductancia al aumentar la resistencia, afectando negativamente la fotosintesis (Huang et al., 1975, Boyer, 1970).

En condiciones de riego a capacidad de campo, la fotosintesis de la quinua muestra valores normales en plantas C3, al ser comparada con Brassica napus L. Jensen et al., 1996), Lupinus consentinii Guss (Jensen et al., 1989), Hordeum distichum L. (Mogensen et al., 1994), Triticum aestivum L. (Weyrich et al., 1995), con $22 \mu_{\text {molm }}{ }^{-2} \mathrm{~s}^{-1}$ en promedio para todo el ciclo de crecimiento.

La conductancia varía en función a cicrtos factores, siendo uno de ellos el estado energético del agua del suelo. A medida que el suclo se deshidrata, la conductancia desciende (Turner, 1991); similarmente en quinua la conductancia disminuye significativamente cuando el contenido de agua del suclo se encuentra por debajo del 50 por ciento. De otra parte, el alto grado de asociación $(r=0,98)$ entre la conductancia y la fotosíntesis, explica las variaciones de ambas variables en condiciones de sequía. Un aspecto relevante es que la alta conductancia observada en la ramificación (el ritmo de sequía se desarrolló con cierta lentitud) con respecto a la floración y llenado de grano, indica que la quinua puede adaptarse con mayor facilidad a condiciones de sequia de desarrollo lento.

El cierre de estomas, en quinua expuesta a sequía, ocurre cuando el potencial hidrico de la hoja estuvo en un nivel ligeramente superior en plantas con riego a capacidad de campo, a 0,9 a -1,1 MPa (Jensen $e$ t al., 2000), y entre -1,5 a -2,0 $\mathrm{MPa}$ (Andersen et al.,1996); a pesar de que el potencial hídrico de la hoja desciende consistentemente, el potencial de presión de la hoja muestra valores positivos, salvo en la fase de llenado de grano en la que toma valores de cero.

Como consecuencia de muy bajos niveles de agua en el suclo, provocados por la sequía, el potencial hídrico de la hoja disminuyó de - $0,7 \mathrm{MPa}$ a - 2,3 $\mathrm{MPa}$ en las fase se ramificación y floración, para disminuir hasta $-3,2 \mathrm{MPa}$ en la fase de llenado de grano. En la fase fenológica de ramificación el potencial osmótico, durante los primeros nueve dias de impuesto el período de sequía, muestra niveles similares a los observados en condiciones de riego a capacidad de campo, con valores promedio de $-1,2 \mathrm{MPa}$, para bajar hasta $-2,6 \mathrm{MPa}$ al final del período de sequía. En la fase de floración de $-1,8$ MPa cayó hasta $-2,6 \mathrm{MPa}$, y en el llenado de grano el potencial osmótico fue aun más negativo de $-3,5 \mathrm{MPa}$ (Jensen et al., 2000).

Las relaciones hidricas de quinua se caracterizan por tener bajos potenciales osmóticos fluctuando entre $-1,0$ y $-1,3$ $\mathrm{MPa}$ en plantas bajo riego; se observa un moderado desarrollo de ajuste osmótico de $0,3 \mathrm{MPa}$ solamente en la fase de ramificación, confirmándose resultados anteriores (Andersen et al., 1996), obtenidos en quinua adaptada a condiciones de clima templado, la fracción del agua del apoplasto estaria próximo al 16 por ciento de acuerdo a estimaciones por el método presión-volumen ( $\Lambda$ dersen et al., 1996).

El nivel de potencial osmótico de quinua es comparable con el de especies monocotiledóneas cultivadas, por ejemplo, en Hordeum distichum L. El potencial osmótico a plena hidratación presenta valores también entre $-1,0$ y $-1,3$ $\mathrm{MPa}$ (Andersen et al., 1991).

Otras plantas cultivadas dicotiledóneas C3, como Lupinus consentinii y Brassica napus L., a plena hidratación, muestran solamente potenciales osmóticos de - $0,7 \mathrm{MPa}$ y -0,9 Mpa respectivamente (Jensen y Henson, 1990; Jensen et al., 1996). La característica de la quinua, de presentar bajos valores de potencial osmótico, puede originar tolerancia al déficit hidrico, lo que se refleja en el mantenimiento de la turgencia durante gran parte de los periodos de sequía, lo que constituiría una evidencia consistente.

El ajuste osmótico observado en la fase de ramifica- 
Nuñez, N. La quinua (Cbenopodium quinod Willd.) alternativa de seguridad alimentaria para zonas desérticas

ción y la no existencia de este mecanismo en las otras fases fenológicas puede entenderse a partir que esta respuesta varía con la ontogenia y la diferencia entre genotipos (Morgan, 1980). El ritmo de desarrollo del estrés también influye. Una tasa de desarrollo lento permite a la planta adaptarse al estrés, mediante la acumulación activa de solutos en las hojas (Turner, 1991).

El área foliar específica (AFE), durante la fase de ramificación, decrece tanto en el control, como en el tratamiento en sequía; diferenciándose este último en forma evidente a partir del octavo día de impuesto el déficit hídrico. Contrariamente, durante la floración los valores del àrea foliar específica se mantienen próximos y con escasa variación entre ambos tratamientos. Al llenado de grano, se observa reducción ligera en el tratamiento bajo déficit hídrico en los cuatro últimos días de sequía.

La relación peso túrgido/peso seco $(\mathrm{Pt} / \mathrm{Ps})$ desciende cn forma paralcla al control, mostrando valores más bajos, desde el noveno dia de iniciado el período de sequía, obscrvándose al final del mismo una diferencia de $0,75 \mathrm{a}$ favor del control. En las fases fenológicas de floración y llenado de grano, las diferencias entre los tratamientos son muy ligeras. Por lo que se postula que en la fase de ramificación la sequía provocó un ligero cambio en el tamaño de las células, lo que muestra que en las otras dos fases fenológicas, no se habrian producido cambios significativos en el tamaño de las células. La expresión de bajas relaciones entre peso túrgido y peso se asocian a plantas resistentes a sequía, como resultado de células pequeñas con paredes celulares engrosadas.

Los efectos de la sequía, sobre variables fisiológicas de quinua variedad Sajama, dieron por resultado, bajos pesos de materia seca en comparación con el tratamiento control. El árca foliar especifica y el contenido de clorofila no sufren reducciones significativas. Valores altos de área foliar y clorofila indican un bajo costo metabólico de mantenimiento del área foliar y, en consecuencia, alta productividad. El contenido de almidón, sacarosa y fructuosa es similar, tanto en el control como en condiciones de sequia. Los niveles de prolina son más altos que en el tratamiento control, respuesta que seria un claro indicador que cualquicr exceso de agua puede afectar negativamente el crecimiento y la partición de materia orgánica (Gonzáles et al., 2009).

\section{Respuestas de la quinua en condiciones de salinidad}

El interés por conocer el comportamiento de la quinua en condiciones de salinidad ha conducido a plantearse varias hipótesis. De esta manera, se estudiaron los efectos de la salinidad sobre la germinación de la semilla. Se experimentó con semillas de las variedades Chucapaca, Kamiri, Ratuqui, Robura, Sajama, Samaranti y Sayaña (de procedencia boliviana), que fueron evaluadas en las siguientes condiciones: a) en soluciones de $\mathrm{NaCl}$ a 0,$05 ; 0,1 ; 0,15$ y $0,2 \mathrm{M}$; b) $\mathrm{pH}$ de 3 - 10; y c) combinación de $\mathrm{NaCl} 0,1$ y $0,2 \mathrm{M}$ con las distintas soluciones de $\mathrm{pH}$. Los efectos del $\mathrm{pH}$ por si mismos no influyen en el porcentaje de germinación; las varie dades Sajama, Sayaña, y Robura, se muestran menos sensibles, a la disminución de la germinación al ser expuestas a la combinación de 0,1 y $0,2 \mathrm{M}$ de $\mathrm{NaCl}$ con $\mathrm{pH}$ de 3-10. Otro resultado de interés es que a $0,1 \mathrm{M}$ de $\mathrm{NaCl}$, todos los $\mathrm{pH}$ probados, activaron la germinación; sin embargo a 0,2 M el efecto fue significativamente menor (Bocro et al., 2001).

Tomando como referencia únicamente la salinidad sobre la germinación, se evaluaron 103 accesiones de la colección representativa de Perú, del banco de germoplasma de la Universidad Nacional del Altiplano de Puno (Perú) y de un total de 1029. En un primer experimento semillas del cultivar peruano 03-21-072RM fueron germinadas en placas petri a $2^{\circ} \mathrm{C}$, en concentraciones salinas de $\mathrm{NaCl}$ de cero hasta $20 \mathrm{dSm}^{-1}$; se encontraron altas tasas de germinación (mayores al $75 \%$ ) en todos los niveles salinos probados (Jacobsen et al., 2001).

Las altas tasas de germinación observadas hasta los $20 \mathrm{dSm}$ (en el primer experimento) llevaron a un segundo experimento, para el tamizado de tolerancia a salinidad, en la colección representativa peruana de quinua de diez cultivares andinos, las que fueron puestas a germinar en tres concentraciones de sal $(0,0 ; 0,5$ y $0,6 \mathrm{M} \mathrm{NaCl})$. De ellas fueron elegidas quince accesiones por su alta tasa de germinación, dos días después de colocadas en la concentración salina más alta. Sin embargo fucron solamente cuatro accesiones las que presentaron hojas cotiledonales, las demás murieron. Una tercera prueba de tamizado de las accesiones más tolerantes, en diez concentraciones de $\mathrm{Nacl}$, en el que a partir de 0,0 hasta $1,5 \mathrm{M}$, sirvió para demostrar que la quinua puede germinar en 24 horas en una solución de $0,3 \mathrm{M} \mathrm{NaCl}$. Los cultivares mejorados de Perú y Bolivia tienen tasas de germinación similares a los cultivares nativos. El cultivar comercial Kancolla (peruano) alcanza un porcentaje de germinación de hasta el $100 \%$ en siete días en una concentración de $0,5 \mathrm{M}$ $\mathrm{NaCl}$, no germina a $0,6 \mathrm{M} \mathrm{NaCl}$. Las semillas de las accesiones más tolerantes demoraron entre seis a siete días para superar el $75 \%$ de germinación en $0,6 \mathrm{M} \mathrm{NaCl}$, el proceso de germinación se inició a los tres días de iniciada la prueba (Jacobsen et al., 2001).

La necesidad de identificar los posibles mecanismos de la tolerancia a la salinidad en plantas de quinua se manifiestan en experimentos como en el que se probaron dos cultivares de quinua con antecedentes de tolerancia a las sales, uno procedente del desierto sur del altiplano boliviano y otro del banco de germoplasma de la Universidad Nacional del Altiplano del Puno (Perú). Al inicio de la formación de las panojas, se aplicaron nueve gradientes de salinidad desde $<1$ hasta $40 \mathrm{mScm}^{-1}$ a $25^{\circ} \mathrm{C}$, utilizando agua de mar diluida. Ias variables, diámetro de tallo e índice de cosecha, muestran mayor grado de tolerancia, con relaciones tratamiento salino/tratamiento control, cercanos a uno, lo que expresa pequeñas variaciones de estas variables con el incremento de salinidad. De manera similar, a especies tolerantes a sales, la conductancia estomatal es muy sensible a la salinidad y, las respuestas, con respecto al área foliar, producción de biomasa, rendimiento de grano e índice de cosecha. La quinua mostró mejores respuestas entre 10 y $20 \mathrm{mScm}^{1}$, en comparación con menores niveles de salinidad, por lo que se le reconoce como una halófita facultativa; sin cmbargo existen diferencias entre cultivares (Jacobsen $t$ al., 2000; Jacobsen et al., 2003).

Entre las respuestas fisiológicas de la quinua al estrés . por sales se encuentra la producción de solutos compatibles como mecanismo para soportar la salinidad y mantener el 
Nuñez, N. La quinua (Cbenopodinm quinoa Willd.) Alternativa de seguridad alimentaria para zonas desérticas.

potencial osmótico. En estudios recientes, se ha encontrado solutos compatibles en hojas cotiledonales a concentraciones de $250 \mathrm{mM}$; sin embargo por los bajos niveles de glicinabetaína y prolina, es probable que estos solutos cumplan un rol secundario en el ajuste osmótico. En cambio en plantas que crecen en medios muy salinos, se ha constatado un incremento de iones inorgánicos y de solutos compatibles, tales como betaina, trehalosa y trigonelina, que son más evidentes a medida que el estrés aumenta; la producción de estos solutos constituyen un mecanismo de la quinua para no sufrir daños irreparables por la salinidad y se encontraron altos contenidos de trigonelina Las quinuas procedentes del altiplano presentan mayor capacidad de acumulación de solutos que las de valle (Morales et al., 2011).

También se demostró que, la quinua además de la producción de solutos compatibles es capaz de acumular sales, mediante mecanismos de retención de agua, tal como la disminución de la conductancia estomatal, sistema radicular capaz de excluir sales y genes que probablemente previenen daños por exceso de sales (Morales et al., 2011).

\section{CONCLUSIONES}

Chenopodium quinoa Willd. presenta respuestas fisiológicas asociadas con la tolerancia a la sequía, como relaciones hídricas con bajos niveles de $\mathrm{Pt} / \mathrm{Ps}$, potencial osmótico, moderado nivel de ajuste osmótico, capacidad para mantener turgencia positiva; el intercambio gaseoso se caracteriza por la reducción de la conductancia. En condiciones de sequía muestra altas tasas de asimilación; los excesos de agua son contraproducentes; lo que se considera como un indicador muy valioso para su cultivo en zonas desérticas. Las respuestas en condiciones de salinidad muestran que posee varios mecanismos que le permiten prosperar en suelos salinos, por lo que se le puede utilizar para mejorar suelos afectados por sales, con ahorro significativo de agua en comparación a otros cultivos colonizadores como la alfalfa.

\section{REFERENCIAS BIBLIOGRÁFICAS}

ALIDE, (2010). Revista N³ de la Asociación Latinoamericana de Instituciones Financieras para el Desarrollo. Julio-Setiembre.

Andersen, S.D., Rasmussen, L., Jensen, C.R., Mogensen, V.O., Andersen, M.N., and Jacobsen, S.E. (1996). Leaf water relations and gas exchange of field grown Cbenopodium quinoa Willd. During drought. In: COST 814. Small grain cereals and psendo-cereals. Workshop at KV L, Copenaghen, Denmark, 22-24 February 1996. Eds. O. Stolen, K. Pithan and J. Hill. pp 117-122. European Commision.

Andersen, M.N., Jensen, C.R., and Loch, R. (1991). Derivation of pressure-volume curves by a non linear regression procedure, and determination of apoplastic water. J. Exp. Bot. 42: 159-165

Boero, C., Gonzáles, J.A., y Prado, F.F. (2001). Germinación de diferentes variedades de quinua (Chenopodium quinoa Willd.) bajo distintas condiciones de salinidad y $\mathrm{pH}$. En: Primertaller Internacional sobre quinua. Proyecto quinua, CIP-DANIDA, UNALM, CIP, UNAP. Ed.
Jacobsen, S.E., Mujica, A. y Portillo, Z. Lima, Perú. pp 287-290.

Boyer, J.S. (1970). Differing sensitivity of photosynthesis to low leaf water potentials in corn and soybean. Plant Pbysiol. 46: 236-239.

Canahua, A. (1977). Observaciones del comportamiento de la quinua a la sequía. En: Actas del primer Congreso Internacional de Cultivos Andinos. Ayacucho, Perú. pp 390-392.

Cárdenas, M. (1944). Descripción preliminar de las variedades de Chenopodium quinoa de Bolivia. Revista de Agricultura. Universidad Mayor de San Simón de Cochabamba. Volumen 2, $\mathrm{N}^{\circ} 02$, pp 13-26.

FAO. (2011). La quinua: Cultivo milenario para contribuir a la seguridad alimentaria mundial. Oficina Regional para América Latina y el Caribe. Roma. 66p.

FAO. (2007). Secuestro de carbono en tierras áridas. Informes sobre recursos mundiales de suelos 102. Roma. 135p.

Gonzáles, J.A., Gallardo, M., Hilal, M., Rosa, M., Prado, F.E. (2009). Physiological responses of quinoa (Chenopoium quinoa Willd.) to drought and waterlogging stresses: dry matter partitioning. Botanicalstudies $50: 35-42$.

Huang, C. Y., Boyer, J. S., and Vanderhef, L.N. (1975). Limitation of acetylene reduction (nitrogen fixation) by photosynthesis in soybean having low water potentials. Plant Plysiol. 56: 228

Jacobsen, S.E. (2003). The Worldwide Potential for Quinoa (Chenopodium quinoa Willd.). Food Reviews International. Vol. 19 pp 167-177.

Jacobsen, S.E., Mujica, A. Jensen, C.R. (2003). The resistance of quinoa (Chenopodium quinoa Willd.) to Adverse Abiotic Factors. Food Reviens International Vol. 19pp 99-109.

Jacobsen, S.E., Quispe, H., Christiansen, J. L., Mujica, A. (2000). What are the mechanisms responsible for salt tolerance in quinoa (Cbenopodium quinoa Willd.)? In: COST action 814. Crop development for de cool and wet regions of Europe. Acivements and future prospects. Proceedings of de final conference. Perdone, Italy $10-13$ may 2000. Eds. G. Parente and J. Frame. pp 511-516. European Commision.

Jacobsen, S.F., Ruiz, F. Mujica, A., Christiansen, J.L. y Ortiz. R. (2001). Evaluación de accesiones de quinua para la tolerancia a la salinidad. En: Primer taller Internacional sobre quinua. Proyecto quinua, CIP-DANIDA, UNALM, CIP, UNAP. Ed. Jacobsen, S.E., Mujica, $A$. y Portillo, Z. Lima, Perú.pp 301-304.

Jacobsen, S.E. y Risi, J. (2000). Distribución geográfica de la quinua fuera de los países andinos. En: Quinua: Chenopodium quinoa Willd. Ancestral cultivo andino, alimento del presente y del futuro. Red de Cooperación en Producción de Cultivos Alimenticios, FAO, UNA. Ed. Mujica, A., Izquierdo, J., Marathee, J. P. y Jacobsen, S.E. Santiago, Chile. pp 56-70.

Jensen, C.R., Jacobsen, S. F., Andersen, M.N., Núñez, N., Andersen, S.D., Rasmusen, L., Mogensen, V.O. (2000). Leaf exchange and water relations Characteristics of field quinoa (Cbenopodium quinoa Willd.) during soil drying. Eur: J. Agron. 13: 11-25. 


\section{Ciencia \& Desarrollo}

Nuñez, N. La quinua (Chenopodium quinoa Willd.) alternativa de seguridad alimentaria para zonas desérticas.

Jensen, C.R. Mogensen, V.O., Mortensen, G., Andersen, M.N., Schjorring, J.K., Thage, J.H., and Koribidis, J. (1996). Leaf photosynthesis and drought adaptation in field grown oilssed rape (Brassica napus L.) Austr. J. Plant Physiol. 23: 631-644.

Jensen, C.R., and Henson, I.E. (1990). Leaf water relations characteristics of Lupinus angustifolius and Lupinus consentinii. Oecologia. 82:114-121.

Larcher, W. (1995). Physiological Plant Ecology. Third Edition. Springer-Verlag. Berlin. Germany. pp 215-379.

Medrano, H., Flexas, J. (2004). Respuestas de las plantas el estrés hídrico En: Ecofisiologia Vegetal. Una Ciencia de Sintesis. Editores Thomson. Madrid, España. pp 253286

Morales, A.J., Bajgain, P., Garver, Z., Maughan, P.J., Udall, J.A. (2011). Physiological responses of Chenopodium quinoa to salt stress. International Journal of Plant Physiology $3(13): 219-232$

Morgan, J.M. (1980). Differences in adaptation to water stress within crop species. In: adaptation of plants to water and high temperature stress. N.C. Turner and
P.J. Kramer. Editors. New York. pp 369-382.

Mujica, A., Izquierdo,J. y Marathee, J.P. (2000). Origen y descripción de la quinua. En: Quinua: Chenopodium quinoa Willd. Ancestral cultivo andino, alimento del presente y del futuro. Red de Cooperación en Producción de Cultivos Alimenticios, FAO, UNA. Ed. Mujica, A., Izquierdo, J., Marathee, J. P. y Jacobsen, S.E. Santiago, Chile. pp 9-29.

Tapia, M. (2001). Zonificación agroecológica del cultivo de la quinua (Chenopodium quinoa Will.). En: Primer taller Internacional sobre quinua. Proyecto quinua, CIPDANIDA, UNALM, CIP, UNAP. Ed. Jacobsen, S.E., Mujica, A. y Portillo, Z. Lima, Perú. pp 17-25.

Turner, N.C. (1991). Measurent and influence plant factor on stomatal conductance in the field. Agricultural and forest meteorology. 54: 137-154.

UNALM. (1982). Zonas áridas $\mathrm{N}^{\circ} 01$. Centro de Investigaciones de Zonas Áridas. PRDCY - OEA. Ed. Velásquez, D. Lima, Perú. 126p.

UNEP. (1997). World atlas desertification. 2nd Edition. Nairobi. 182 p.
Correspondencia:

Nivardo Nuñez Torreblanca: ntor_01@hotmail.com
Fecha de Recepción: 31/03/2014

Fecha de Aceptación: 05/06/2015 